\title{
Transcranial direct current stimulation for Alzheimer disease
}

\author{
Antonio Gangemi, Rosa Angela Fabio
}

\begin{abstract}
Background. Alzheimer disease (AD) is the most common form of senile dementia, with an incidence of $1 \%$ to $3 \%$ in the general population. Currently, there is no cure for $\mathrm{AD}$, and treatments are mainly drug-based. Transcranial direct current stimulation (tDCS) is a non-invasive method to induce brain plasticity. This study aimed to investigate the effects of 10 days of tDCS over the left frontotemporal cortex on cognitive and neurophysiological functioning in AD patients.

Methods. 26 patients with $\mathrm{AD}$ aged 70 to 85 years were randomised to receive tDCS of the left frontotemporal cortex $(n=15)$ or sham $t D C S$ $(n=11)$. Cognitive and neurophysiological functions were assessed before and after tDCS using the Milan Overall Dementia Assessment and electroencephalography (for alpha and beta bands and P300 latency and amplitude).

Results. tDCS resulted in increased cognitive functions in terms of temporal orientation, spatial orientation, reversal learning, verbal intelligence, story, production of word, and attention, as well as increased beta band and decreased P300 latency.
\end{abstract}

Conclusion. tDCS is simple, safe, inexpensive, and non-invasive method to enhance cognitive functions in patients with AD.

Key words: Alzheimer disease; Electroencephalography; Transcranial direct current stimulation
Department of Clinical and Experimental Medicine, University of Messina, Italy

Correspondence to: Rosa Angela Fabio, Department of Clinical and Experimental Medicine, University of Messina, via Bivona, Messina, Italy.Email: rafabio@unime.it

\section{INTRODUCTION}

In Italy in $2018,35 \%$ of the population were aged $\geq 65$ years, and the life expectancy was 80.8 years for men and 85.2 years for women. Alzheimer disease (AD) is the most common form of senile dementia, affecting $10 \%$ of people older than 65 years and nearly $50 \%$ of those older than 85 years, with an incidence of $1 \%$ to $3 \%$ in the general population. It is a chronic illness with long preclinical and prodromal phases (20 years) and an average disease duration of 8 to 10 years. AD is a neurodegenerative disorder characterised by a gradual decline in numerous cognitive processes. ${ }^{1,2}$ On neuroimaging, $\mathrm{AD}$ is associated with pathological and structural changes in the brain, especially in the temporal cortex. $^{3}$ On quantitative electroencephalography,
$\mathrm{AD}$ is characterised by increased theta power, decreased alpha and beta power, and decreased coherence in the alpha and theta band in posterior regions. ${ }^{4}$ These abnormalities are associated with functional disconnections of cortical areas, death of cortical neurons, axonal pathology, and cholinergic deficits, resulting in dysfunction of specific cortical areas. $^{4}$

$\mathrm{AD}$ is the result of accumulation of amyloid plaques and neurofibrillary tangles. Amyloid plaques are located between neurons as dense clusters of beta-amyloid molecules, a sticky type of protein that clumps together. Amyloid plaques cause tiny filaments called microtubules to twist and disrupt the transportation of nutrients and organelles. Neurofibrillary tangles are found inside the neurons 
of the brain and are caused by defective tau proteins that clump into a thick, insoluble mass.

AD behavioural effects are caused by changes in neuronal activity (changes in modulatory transmitter systems and network connectivity) secondary to the disease process; therapies that address these changes have been reported. . $^{5-10}$

Transcranial direct current stimulation (tDCS) is a simple, safe, inexpensive, and non-invasive method of brain stimulation. Small electrodes are placed on the scalp above the involved area. tDCS induces prolonged functional changes in the cerebral cortex through synaptic and non-synaptic mechanisms and facilitates cortical excitability and hence neuroplasticity., ${ }^{911-15}$ tDCS over the frontotemporal area improves memory in patients with $\mathrm{AD} \cdot{ }^{11,15}$ tDCS of the temporal cortex enhances name recall in healthy elderly persons ${ }^{16}$ and improves recognition memory in patients with AD. ${ }^{17,18}$ tDCS improves the performance of recognition tasks in patients with AD. ${ }^{12}$ However, evidence remains insufficient to support tDCS as an intervention for AD; placebocontrolled trials are thus warranted to assess the efficacy of tDCS of the temporal cortex in patients with AD. This study aimed to investigate the effects of 10 days of $\mathrm{tDCS}$ over the left frontotemporal cortex on cognitive and neurophysiological functioning in $\mathrm{AD}$ patients.

\section{METHODS}

The study was conducted according to the Declaration of Helsinki and approved by the university research ethics committee (Reference: 2016-36). Informed consent was given by patients and their caregivers. The definition of $\mathrm{AD}$ was based on the National Institute of Neurological Communicative Disorders and Stroke, the Alzheimer disease and Related Disorders Association, and the Diagnostic and Statistical Manual of Mental Disorders version V. Patients with a Mini-Mental State Examination score of 12 to 25 (adjusted to the level of education) were included. Those with other neuropsychiatric diseases were excluded. 26 patients with $\mathrm{AD}$ aged 70 to 85 years were randomised to receive tDCS of the left frontotemporal cortex $(n=15)$ or sham $\mathrm{tDCS}(\mathrm{n}=11)$ [TABLE 1]. All patients were under treatment with cholinesterase inhibitors, and the dosage was the same during the study.
For tDCS, direct current was transferred via a saline-soaked pair of surface sponge electrodes (35 $\left.\mathrm{cm}^{2}\right)$ by a battery-driven, constant-current stimulator. According to the 10-20 international system for electroencephalographic electrodes placement, the dorsolateral prefrontal cortex was stimulated with the anode electrode placed over F3F7, and the left temporal cortex was stimulated with an anode electrode placed over F7. The reference cathode electrode was placed over the right supraorbital area. The dorsolateral prefrontal cortex is associated with working memory performance, according to neuroimaging, repetitive transcranial magnetic stimulation, and tDCS studies. Patients and examiners were blind to the type of tDCS.

Cognitive function was assessed before and after tDCS using the Milan Overall Dementia Assessment (MODA). ${ }^{19,20}$ The test-retest reliability of MODA was $0.83 .{ }^{20}$ MODA comprises one behavioural scale and two test sections. The behavioural scale measures five activities of daily living: walking, dressing, personal hygiene, control of sphincters, and eating. Answers are sought from a close relative. Scores for each aspect range from 0 (in need of total supervision) to 3 (total autonomy), with an overall score of 0 to 15 .

The first test section assesses (1) temporal orientation (5 questions on the day of the week, the date of the month, the month, the year, and the time of day, with scores range from 0 to 10), (2) spatial orientation (3 questions on topographical information on the town and country, with scores range from 0 to 3), (3) personal orientation (7 questions on personal background, with scores range from 0 to 10 . Standardised items are weighted differently according to face value difficulty), and (4) family orientation (12 questions on name and age of four different family members, and whether they are alive or dead, with scores range from 0 to 12). Total scores range from 0 to 35 .

The second test section is based on an Italian standardised series of neuropsychological tests and assesses attention (digit cancellation, reversal learning), intelligence (logical reasoning), memory (prose memory), language (verbal comprehension, fluency), space cognition (finger agnosia, constructional apraxia), and visual perception (figure completion, street test). Only the easiest items were 
TABLE 1

Demographic data of 26 patients with Alzheimer disease

\begin{tabular}{ccccc}
\hline Patient & Sex/ & Education, & Mini Mental & Disease \\
age, y & $y$ & State & duration, \\
& & & $\begin{array}{c}\text { Examination } \\
\text { score }\end{array}$ & $y$
\end{tabular}

Transcranial direct

current stimulation

(tDCS) group $(n=15)$

\begin{tabular}{|c|c|c|c|c|}
\hline 1 & $\mathrm{M} / 72$ & 5 & 22 & 3 \\
\hline 2 & $\mathrm{M} / 70$ & 8 & 18 & 4 \\
\hline 3 & M/83 & 5 & 18 & 3 \\
\hline 4 & $\mathrm{~F} / 76$ & 10 & 23 & 1 \\
\hline 5 & $\mathrm{M} / 81$ & 3 & 22 & 1 \\
\hline 6 & $\mathrm{~F} / 71$ & 8 & 20 & 2 \\
\hline 7 & $\mathrm{~F} / 79$ & 5 & 20 & 3 \\
\hline 8 & $\mathrm{M} / 77$ & 5 & 19 & 2 \\
\hline 9 & $\mathrm{~F} / 72$ & 7 & 22 & 3 \\
\hline 10 & $M / 69$ & 8 & 21 & 3 \\
\hline 11 & M/68 & 8 & 20 & 2 \\
\hline 12 & $\mathrm{M} / 71$ & 8 & 19 & 3 \\
\hline 13 & $\mathrm{~F} / 73$ & 7 & 21 & 2 \\
\hline 14 & $\mathrm{M} / 75$ & 8 & 20 & 1 \\
\hline 15 & $\mathrm{~F} / 73$ & 8 & 17 & 2 \\
\hline \multicolumn{5}{|c|}{$\begin{array}{l}\text { Sham tDCS group } \\
(n=11)\end{array}$} \\
\hline 1 & F/69 & 5 & 20 & 3 \\
\hline 2 & $\mathrm{~F} / 70$ & 8 & 18 & 4 \\
\hline 3 & $\mathrm{~F} / 80$ & 5 & 19 & 3 \\
\hline 4 & $\mathrm{~F} / 76$ & 8 & 21 & 1 \\
\hline 5 & $\mathrm{M} / 81$ & 4 & 22 & 2 \\
\hline 6 & $\mathrm{~F} / 70$ & 8 & 20 & 2 \\
\hline 7 & $\mathrm{M} / 77$ & 5 & 20 & 1 \\
\hline 8 & $\mathrm{M} / 77$ & 5 & 19 & 1 \\
\hline 9 & $\mathrm{M} / 71$ & 7 & 22 & 2 \\
\hline 10 & $\mathrm{~F} / 80$ & 8 & 21 & 1 \\
\hline 11 & $\mathrm{M} / 74$ & 8 & 21 & 2 \\
\hline
\end{tabular}

chosen to avoid a 'floor performance' in demented patients. Total scores range from 0 to 50 .

In addition, the effects of tDCS on beta and alpha bands and on P300 latency and amplitude were evaluated using electroencephalography. P300 reflects attention, stimulus evaluation, judgment, and decision-making. P300 latency increases in patients with $\mathrm{AD}$ compared with those without $\mathrm{AD}, 5$ but there is no consensus on P300 amplitude.
Statistical analysis was performed using SPSS (Windows version 22; IBM Corp, Armonk [NY], US). Level of significance was set at $\alpha=0.05$. The Kolmogorov-Smirnov test was used to ascertain normal distribution of data. The tDCS and sham tDCS groups were compared in terms of MODA scores. Repeated-measures analysis of variance of between-group (group) and within-group (phase and subscale) factors were performed to detect the effects of time on dependent variables. Bonferroni corrected $t$ tests were used for post-hoc analysis $(p<0.01)$.

\section{RESULTS}

There was no adverse effect associated with tDCS. Repeated-measures analysis of variance showed that the effect of group was not significant, but the effect of the phase $x$ group interaction was significant $(\mathrm{F}(1,24)=12.98, \mathrm{p}<0.001)$, as was the effect of time $\times$ phase $\times$ subscale interaction $(F(1,24)=12.96, p<0.01)$ [TABLE 2]. This indicated that sham tDCS resulted in a stable trend over time, whereas tDCS resulted in an increase trend in some subscales over time. Posthoc analysis was conducted separately for the two groups.

From pre-test to post-test, tDCS significantly improved cognitive functions in terms of temporal orientation $(\mathrm{t}(14)=1.99, \mathrm{p}<0.05)$, spatial orientation $(\mathrm{t}(14)=2.01, \mathrm{p}<0.05)$, reversal learning $(\mathrm{t}(14)=2.87$, $\mathrm{p}<0.01)$, verbal intelligence $(\mathrm{t}(14)=2.19, \mathrm{p}<0.04)$, story test $(\mathrm{t}(14)=2.89, \mathrm{p}<0.01)$, production of word $(\mathrm{t}(14)=2.23, \mathrm{p}<0.04)$, and attention $(\mathrm{t}(14)=1.99$, $\mathrm{p}<0.05)$. However, sham tDCS resulted in significantly decreased cognitive functions in terms of temporal orientation $(\mathrm{t}(9)=2.51, \mathrm{p}<0.05)$, verbal intelligence $(\mathrm{t}(10)=2.25, \mathrm{p}<0.05)$, and street test $(\mathrm{t}(10)=2.89, \mathrm{p}<0.05)$.

The effect of time $\times$ phase interaction was significant in P300 latency $(F(1,24)=7.16 ; p<0.01$, TABLE 3). This indicated that only tDCS resulted in decreased P300 latency. In addition, from pre-test to post-test $\mathrm{tDCS}$ resulted in increased beta band $(\mathrm{t}(14)=3.34, \mathrm{p}<0.01$, TABLE 3$)$.

\section{DISCUSSION}

tDCS resulted in increased cognitive functions in terms of temporal orientation, spatial orientation, reversal learning, verbal intelligence, story test, 
TABLE 2

Milan Overall Dementia Assessment (MODA) subscale scores before and after transcranial direct current stimulation (tDCS)

\begin{tabular}{lccccc}
\hline MODA subscale & \multicolumn{2}{c}{ Pre-test } & & \multicolumn{2}{c}{ Post-test } \\
\cline { 2 - 3 } \cline { 5 - 6 } & tDCS group & Sham tDCS group & & tDCS group & Sham tDCS group \\
\hline Temporal orientation & $4.20 \pm 1.373$ & $3.60 \pm 0.699$ & & $4.87 \pm 1.187$ & $2.70 \pm 0.675$ \\
Spatial orientation & $2.27 \pm 0.799$ & $2.00 \pm 0.471$ & & $2.80 \pm 0.862$ & $1.90 \pm 0.316$ \\
Personal orientation & $8.53 \pm 0.919$ & $8.20 \pm 0.919$ & & $8.07 \pm 0.961$ & $7.50 \pm 0.850$ \\
Family orientation & $8.60 \pm 1.056$ & $9.40 \pm 1.174$ & & $8.73 \pm 2.086$ & $8.70 \pm 0.949$ \\
Autonomies & $11.80 \pm 1.740$ & $12.30 \pm 1.337$ & & $11.07 \pm 1.831$ & $11.70 \pm 1.059$ \\
Reversal learning & $3.53 \pm 0.743$ & $3.90 \pm 0.876$ & & $4.20 \pm 0.676$ & $3.70 \pm 0.823$ \\
Verbal intelligence & $3.80 \pm 1.146$ & $4.40 \pm 1.075$ & & $4.53 \pm 0.990$ & $3.20 \pm 1.033$ \\
Story test & $3.60 \pm 0.986$ & $3.90 \pm 0.876$ & & $4.27 \pm 0.594$ & $3.50 \pm 1.080$ \\
Production words & $3.27 \pm 1.438$ & $3.10 \pm 0.876$ & & $2.60 \pm 1.454$ & $2.90 \pm 0.919$ \\
Token test & $4.87 \pm 0.516$ & $4.60 \pm 0.516$ & & $4.60 \pm 910$ & $4.30 \pm 0.949$ \\
Digital agnosia & $3.53 \pm 0.990$ & $3.10 \pm 1.370$ & & $3.47 \pm 0.915$ & $2.90 \pm 1.197$ \\
Constructive apraxia & $2.73 \pm 0.799$ & $2.10 \pm 0.994$ & & $2.73 \pm 0.594$ & $1.80 \pm 0.789$ \\
Street test & $2.60 \pm 0.632$ & $2.00 \pm 0.943$ & & $2.60 \pm 0.632$ & $1.80 \pm 0.789$ \\
Attentional test & $8.20 \pm 1.320$ & $8.50 \pm 0.850$ & & $8.93 \pm 0.799$ & $8.20 \pm 0.789$ \\
\hline
\end{tabular}

TABLE 3

Alpha and beta bands and P300 latency before and after transcranial direct current stimulation (tDCS)

\begin{tabular}{lccccc}
\hline \multirow{2}{*}{ Test } & \multicolumn{2}{c}{ Pre-test } & & \multicolumn{2}{c}{ Post-test } \\
\cline { 2 - 3 } \cline { 5 - 6 } & tDCS group & Sham tDCS group & & tDCS group & Sham tDCS group \\
\hline Alpha band & $8.3 \pm 0.403$ & $8.3 \pm 0.411$ & & $8.5 \pm 0.435$ & $8.1 \pm 0.582$ \\
Beta band & $13.3 \pm 1.6$ & $13.5 \pm 1.4$ & & $15.4 \pm 1.0$ & $13.4 \pm 1.5$ \\
P300 latency & $351.4 \pm 28.9$ & $358.6 \pm 30.6$ & & $332.6 \pm 21.7$ & $356.9 \pm 32.6$ \\
\hline
\end{tabular}

production of word, and attention, as well as increased beta band and decreased P300 latency. Our results differed from those reported no increase in memory performance after tDCS. ${ }^{21}$ This may be because our patients had less severe $\mathrm{AD}$ and thus were more receptive to $\mathrm{tDCS}$, which is less effective in advanced stages of AD with reduced neuroplasticity (long-term potentiation). ${ }^{21}$ Nonetheless, our results are in accordance with other studies., ${ }^{911,12,14,15}$ For example, one study reported increased visual recognition memory scores by $8.9 \%$ at 1 month after 5 consecutive days of 30-minute tDCS of the temporal cortex in patients with $\mathrm{AD} .{ }^{11}$

One limitation of the present study was the small sample size, and thus interpretation of findings should be cautious. Large-scale randomised controlled studies are warranted. In addition, comparison of different levels of severity of $\mathrm{AD}$ is necessary, as $\mathrm{AD}$ is associated with reduced neuroplasticity (long-term potentiation), which is inversely associated with severity. ${ }^{21}$ In addition, there may have been test-retest discrepancy of MODA. In our study, MODA scores showed no coherence, although a study of $186 \mathrm{AD}$ patients reported adequate $(r=0.83)$ test-retest reliability of MODA. ${ }^{22}$ Thus, it is necessary to compare MODA with different cognitive tests to assess the level of coherence between different cognitive processes. Longitudinal follow-up studies are warranted to assess whether cognitive functions after tDCS increase with time, and to what extent severity of 
$\mathrm{AD}$ is predictive of levels of neuroplasticity. Further studies are needed to determine the role of tDCS in treatment of $\mathrm{AD}$ and what professional support is needed for this increasing patient group.

\section{CONCLUSION}

tDCS is simple, safe, inexpensive, and non-invasive method to enhance cognitive functions in patients with AD.

\section{DECLARATION}

The authors had no affiliations with or involvement in any organisation or entity with any financial interest (such as honoraria, educational grants, membership, employment, consultancies, stock ownership, other equity interest, expert testimony, or patent-licensing arrangements), or non-financial interest (such as personal or professional relationships, affiliations, knowledge or beliefs) in the subject matter or materials discussed in this manuscript.

\section{REFERENCES}

1. Petersen RC, Parisi JE, Dickson DW, Johnson KA, Knopman DS, Boeve BF, et al. Neuropathologic features of amnestic mild cognitive impairment. Arch Neurol 2006;63:665-72. Crossref

2. Galvin JE, Roe CM, Powlishta KK, Coats MA, Muich SJ, Grant E, et al. The AD8: a brief informant interview to detect dementia. Neurology 2005;65:559-64. Crossref

3. Blennow K, de Leon MJ, Zetterberg H. Alzheimer's disease. Lancet 2006;368:387-403. Crossref

4. Marceglia S, Mrakic-Sposta S, Rosa M, Ferrucci R, Mameli F, Vergari $\mathrm{M}$, et al. Transcranial direct current stimulation modulates cortical neuronal activity in Alzheimer's disease. Front Neurosci 2016;10:134. Crossref

5. Pedroso RV, Fraga FJ, Corazza DI, Andreatto CA, Coelho FG, Costa JL, et al. P300 latency and amplitude in Alzheimer's disease: a systematic review [in English, Portuguese]. Braz J Otorhinolaryngol 2012;78:126-32. Crossref

6. Fabio RA, Billeci L, Crifaci G, Troise E, Tortorella G, Pioggia G. Cognitive training modifies frequency EEG bands and neuropsychological measures in Rett syndrome. Res Dev Disabil 2016;53-54:73-85. Crossref

7. Fabio RA, Caprì T, Mohammadhasani N, Gangemi A, Gagliano A, Martino G. Frequency bands in seeing and remembering: comparing ADHD and typically developing children. Neuropsychol Trends 2018;24:97-116. Crossref

8. Fabio RA, Castelli I, Marchetti A, Antonietti A. Training communication abilities in Rett Syndrome through reading and writing. Front Psychol 2013;4:911. Crossref
9. Fabio RA, Gangemi A, Capri T, Budden S, Falzone A. Neurophysiological and cognitive effects of transcranial direct current stimulation in three girls with Rett Syndrome with chronic language impairments. Res Dev Disabil 2018;76:7687. Crossref

10. Fabio RA, Giannatiempo S, Oliva P, Murdaca AM. The increase of attention in Rett Syndrome. A pre-test/post-test research design. J Dev Phys Disabil 2011;23:99-111. Crossref

11. Boggio PS, Ferrucci R, Mameli F, Martins D, Martins O,Vergari M, et al. Prolonged visual memory enhancement after direct current stimulation in Alzheimer's disease. Brain Stimul 2012;5:22330. Crossref

12. Boggio PS, Khoury LP, Martins DC, Martins OE, de Macedo EC, Fregni F. Temporal cortex direct current stimulation enhances performance on a visual recognition memory task in Alzheimer disease. J Neurol Neurosurg Psychiatry 2009;80:444-7. Crossref

13. Fabio RA, Martinazzoli C, Antonietti A. Costruzione e standardizzazione dello strumento "R.A.R.S." (Rett Assessment Rating Scale) [in Italian]. Ciclo evolutivo e disabilità 2005:825781.

14. Gangemi A, Caprí T, Fabio RA, Puggioni P, Falzone AM, Martino G. Transcranial direct current stimulation (TDCS) and cognitive empowerment for the functional recovery of diseases with chronic impairment and genetic etiopathogenesis. Adv Genet Res 2018:1-18.

15. Ferrucci R, Mameli F, Guidi I, Mrakic-Sposta S, Vergari M, Marceglia S, et al. Transcranial direct current stimulation improves recognition memory in Alzheimer disease. Neurology 2008;71:493-8. Crossref

16. Ross LA, McCoy D, Coslett HB, Olson IR, Wolk DA. Improved proper name recall in aging after electrical stimulation of the anterior temporal lobes. Front Aging Neurosci 2011;3:16. Crossref

17. Cotelli M, Manenti R, Petesi M, Brambilla M, Cosseddu M, Zanetti $\mathrm{O}$, et al. Treatment of primary progressive aphasias by transcranial direct current stimulation combined with language training. J Alzheimers Dis 2014;39:799-808. Crossref

18. Gervits F, Ash S, Coslett HB, Rascovsky K, Grossman M, Hamilton $\mathrm{R}$. Transcranial direct current stimulation for the treatment of primary progressive aphasia: an open-label pilot study. Brain Lang 2016;162:35-41. Crossref

19. Spinnler H,Tognoni G. Standardizzazione e taratura italiana di test neuropsicologici : gruppo italiano per lo studio neuropsicologico dell'invecchiamento [in Italian]. Italian J Neurol Sci 1987;6(Suppl 8):44-6.

20. Brazzelli M, Capitani E, Della Sala S, Spinnler H, Zuffi M. A neuropsychological instrument adding to the description of patients with suspected cortical dementia: the Milan overall dementia assessment. J Neurol Neurosurg Psychiatry 1994;57:1510-7. Crossref

21. Bystad M, Grønli O, Rasmussen ID, Gundersen N, Nordvang $\mathrm{L}$, Wang-Iversen $\mathrm{H}$, et al. Transcranial direct current stimulation as a memory enhancer in patients with Alzheimer's disease: a randomized, placebo-controlled trial. Alzheimers Res Ther 2016;8:13. Crossref

22. Cilia S, Coppola R, Picconi O, Ferrazza P, Surdo V, Tripi G, et al. L'uso del Milan Overall Dementia Assessment (MODA) per individuare il rischio di sviluppo di demenza in soggetti cognitivamente intatti al Mini Mental State Examination (MMSE) [in Italian]. Life Span Disabil 2008;11:151-62. 